E3S Web of Conferences 2, 03002 (2014)

DOI: $10.1051 /$ e3sconf/ 20140203002

(C) Owned by the authors, published by EDP Sciences, 2014

\title{
Future Scenarios Of Urban Mobility And Their Sustainability
}

\author{
Ennio Cascetta \\ University of Naples, Department of Civil, Architectural and Environmental Engineering, Via \\ Claudio, 25, 80125 Napoli, Italy
}

\begin{abstract}
At this point in time transportation is a problem in most cities because it fails to achieve the standards of economic and environmental efficiency that would be desirable in the opinion of the experts, as well as for their citizens and institutions. This is true for cities both in the so-called developed and developing worlds even though individual cities have very different levels of mobility supply and performances. Thinking about future urban mobility is thus not only a challenging intellectual exercise, but could provide some insight on what "evolution trajectories" are to be preferred, or avoided, from the social and economic points of view.
\end{abstract}

\section{The relevance of urban mobility}

The role of mobility in future cities can hardly be overestimated. As urbanized population of the world passed the "symbolic threshold" of 50 percent in 2007 going to exceed $60 \%$ by 2030 and the role of cities in the production of both economic and intellectual value is increasing (only 600 urban centers generate about 60 percent of global GDP; McKinsey Global Institute, 2011), the availability of transportation systems able to accommodate for future mobility needs of people and goods without consuming resources beyond acceptable levels is quintessential. As a matter of fact the quality of the urban environment is increasingly seen as a major competitive factor of cities, e.g. Rogerson 1999. The ease and "environmental footprint" of their mobility systems is one of the key factors contributing to such a quality (Kenworthy, 2006; Banister, 1996).

At this point in time transportation is a problem in most cities exactly because it fails to achieve the standards of economic and environmental efficiency that would be desirable in the opinion of the experts, as well as for their citizens and institutions. This is true for cities both in the so-called developed and developing worlds even though individual cities have very different levels of mobility supply and performances.

Thinking about future urban mobility is thus not only a challenging intellectual exercise, but could provide some insight on what "evolution trajectories" are to be preferred, or avoided, from the social and economic points of view.

\section{The future is rooted in the past}

The future of urban mobility is certainly dependent on how urban transportation systems, and more generally cities, are today. And they are very different among each other in the first place. Cities around the world have different dimensions and structure, have reached solutions to their mobility needs that are very diverse: from pedestrian cities (Venice) to transit cities (e.g. Tokyo) on to cities 
that are entirely dependent on individual modes and mostly the car (e.g. Los Angeles), with all the range in between. Table 1 synthesize the differences in structure and modal shares in cities across continents (Kenworthy and Laube, 2001).

TABLE 1

Urban form in a global sample of cities, 1995

$\begin{array}{lccccccccccc} & \text { CHN } & \text { LIA } & \text { LAM } & \text { AFR } & \text { MEA } & \text { EEU } & \text { WEU } & \text { HIA } & \text { ANZ } & \text { USA } & \text { CAN } \\ \text { Urbandensity } & 146 & 204 & 75 & 60 & 119 & 53 & 55 & 150 & 15 & 15 & 26 \\ \begin{array}{l}\text { (persons/ha) } \\ \begin{array}{l}\text { Proportion of } \\ \text { jobs in CBD }\end{array}\end{array} & 51 & 17 & 29 & 15 & 13 & 20 & 19 & 19 & 15 & 9 & 16 \\ \end{array}$

Private mobllity and prlvate transport Infrastructure In a global sample of cltles, 1995

\begin{tabular}{|c|c|c|c|c|c|c|c|c|c|c|c|}
\hline Travel & $\mathrm{CHN}$ & LIA & LAM & AFR & MEA & EEU & WEU & HIA & ANZ & USA & CAN \\
\hline \multicolumn{12}{|c|}{$\begin{array}{l}\text { Car passenger kilometres } \\
\text { per capita }\end{array}$} \\
\hline & 814 & 1,855 & 2,862 & 2,652 & 3,262 & 2,907 & 6,202 & 3,614 & 11,387 & 18,155 & 8,645 \\
\hline \multicolumn{12}{|c|}{$\begin{array}{l}\text { Motor cycle passenger } \\
\text { kilometres per capita }\end{array}$} \\
\hline & 289 & 684 & 104 & 57 & 129 & 19 & 119 & 357 & 81 & 45 & 21 \\
\hline \multicolumn{12}{|c|}{$\begin{array}{l}\text { Non-motorized modes } \\
\text { ( } \% \text { daily trips) }\end{array}$} \\
\hline & 65 & 32 & 31 & 42 & 27 & 26 & 31 & 28 & 16 & 8 & 10 \\
\hline \multicolumn{12}{|c|}{$\begin{array}{l}\text { Motorized public modes } \\
\text { (\% daily trips) }\end{array}$} \\
\hline & 19 & 32 & 34 & 26 & 18 & 47 & 19 & 30 & 5 & 3 & 9 \\
\hline
\end{tabular}

Source: Kenworthy, J., and F. Laube (2001), The Millennium Cities Database for Sustainable Transport, CD-ROM database, International Union of Public Transport (UITP), Brussels, and Institute for Sustainability and Technology Policy (ISTP), Perth.

The abbreviations are as follows: CHN - Chinese cities; LIA - Low-Income Asian cities; LAM - Latin American cities; AFR African cities; MEA - Middle Eastern cities; EEU - Eastern European cities; WEU - Western European cities; HIA - HighIncome Asian cities; ANZ - Australia/New Zealand cities; USA - American cities; CAN - Canadian cities.

However there are a number of similarities among urban transportation of most cities. The building blocks of their transportation systems are very similar across cities and they differ in the way these components are used. We have different "pure" modes and related technologies to move people (walking, biking, motorized two-wheelers, cars, individual and collective taxis, buses, trams, metros, regional railways) and freight (hand carriers, small vans, medium and large size trucks) and several combinations of them (e.g. park and ride). We have different management arrangements (self production, production by companies operating in exclusive rights, licensing, competition in the market). We have different prices and pricing systems (e.g. congestion pricing, social tickets, oil and energy prices). We have different regulations (e.g. demand management schemes, delivery times etc). We have different technologies for monitoring the system components, to control them, to inform the general public and travelers). Wandering about how urban transportation will be in the next two/three decades means figuring out how the components of their systems will change over this time span.

The future is rooted in the past, but we cannot predict it judging from the past as we cannot know the exact shape of a tree only by observing its roots. 
Changes in complex systems, and urban transportation systems are certainly complex, are of two types: incremental changes and "revolutions".

The first type is related to changes that derive from modifications, either technological and/or organizational, of elements and their arrangements already existing. In my opinion the changes of urban transportation systems over the last 30-40 years are of this type. Basically the components of present systems were already available in the seventies and the eighties, from cars to transit systems, from metro to street cars, from parking arrangements to traffic lights, from traffic and congestion management schemes to radio and TV information on traffic conditions. Obviously since then things have changed a lot new technologies under many respects. Car engines and dimensions, the growth and diffusion of Intelligent Transportation Systems, specially information connected to personal computing first and to smart phones lately, new aesthetic dimension of rail and metro stations (the socalled station renaissance), new regulations on air quality and congestion reduction policies. In some cases things have even gone "backward" with the return of mobility solutions that thirty years ago seemed old fashioned and increasingly marginal such as metro and surface mass transit systems or the comeback of the bike, either electrically powered or not. Certainly transportation in Los Angeles today is closer to what it was in 1984 than to the future then imagined in "Blade Runner" for the year 2014 with flying cars and the rest.

On the other hand revolutions are significant and fast changes induced either by technology and/or organizational innovations. Gilbert and Perl (2010), in their book "Transport Revolutions", define transport revolutions as "a substantial change in a society's transportation activity that occurs in less than 25 years", where substantial change is intended as "either something that was happening before increases or decreases dramatically, say by 50 percent, or a new means of transport becomes prevalent to the extent that it becomes a part of the lives of ten percent or more of the society's population".

Urban transportation in North American cities underwent a revolution in the first three decades of the last century with the widespread use of individual cars, as most European and non European cities saw their transportation systems similarly revolutionized in the decades following WW II. Urban transportation was also revolutionized in a few decades during the second half of the XIX century as railways spread and the dimension and shape of cities changed accordingly.

\section{The drivers of change}

Changes in urban transportation can be induced by a number of factors either acting independently or jointly to shape future configurations. Also, as stated previously, different cities will have different evolution trajectories as their present systems are different and the changes will differ as well.

In the following I will try to list some of the factors that will likely play a role in this process across several cities in the world with some preliminary comments.

\subsection{Socioeconomic factors}

\subsubsection{Land-use and shape of cities}

A few decades is a short time to change significantly the shape of cities, especially the one with a long history in developed Countries. Significant changes in densities and land use are possible though. Cities could keep expanding towards more low density peripheries, increasing the dependence on cars and individual transport modes, or could re-polarize, especially around high capacity transport systems such as rail. Also relationships among cities could change significantly evolving towards systems of cities functionally connected, giving rise to inter-urban daily systems, especially for those cities connected by High Speed Railways in less than 2/3 hours. This type of evolution would further increase the difference among rail-based cities and non rail-based ones. 


\subsubsection{Demographics}

Aging of the population and sustained immigration for most developed cities will likely occur and pose new demands on transportation.

\subsubsection{Activity participation, lifestyles and consumption models}

Participation in e-activities could change the structure of travel demand. So far this participation was below the levels expected one or two decades ago for various reasons and substitution effects with respect to mobility were not that significant.

Another trend potentially relevant to urban mobility is related to the demand of more health conscious mobility styles including larger shares of "muscular modes" such as walking, cycling and assisted cycling. This trends are likely be more significant for medium-sized cities, but the success of bike sharing in large cities such as Paris, could point towards a larger potential.

Wider spread of e-commerce will stress the city logistics with increased demand for parcels distribution and related congestion problems.

Changes in lifestyles and activity participation could modify travel demand, increasing more complex trip patterns, time of the day distribution and require more articulated mobility services.

\subsubsection{Income levels and distributions}

Reduced income inequalities would generate increases in urban travel demand (income elasticity is higher for lower income levels) while the opposite would be true for increasing inequalities, given the same level of overall income. It's possible that the two different trends will show in developing and developed Countries, going in the opposite direction from the last century.

\subsubsection{City governance and citizen participation}

The way in which cities will be governed will certainly influence their mobility system. Increases in participation possibly fostered by the spread of ICT, will increase the awareness of citizens and other stakeholders on problems (e.g. pollution levels, congestion, differentials in accessibility) as well as the effect of proposed policies. The approach to urban decision making, and mobility and transport in particular, will move from the DAD (Decide, Announce and Defend) to the IPADD (Inform, Propose, Audit, Discuss and Decide) style (Cascetta, 2012). The effect are likely to be more visible for those cities where present participation levels are currently low would require different approaches to design and decision making of transport policies.

\subsection{Energy and Power sources}

\subsubsection{Oil and traditional power sources (including bio fuels) availability and prices}

This could be one of the major drivers of change. Two possible scenarios here. One related to decreases of extraction quantities (peaking) and fuel prices with a "forced" transition to alternative energy sources and possibly to more energy efficient travel modes. The other is based on sustained extraction capabilities and/or new technologies (e.g. shale) and keeping the status quo.

\subsubsection{Renewable energy sources, availability and prices}

The main changes could derive from a more than incremental change in renewable energy production costs and storage capacity. 
3.3 Technological innovation in existing transportation modes (dimensions, terminals, control systems, power systems)

\subsubsection{Cars}

Specialization of city cars and improved control and information technologies allowing increased traffic capacity for existing road systems, possibly more refined pay-per-use systems. Diffusion/requirement of vehicle specific remote recognition devices (electronic number plate).

\subsubsection{Trains and metros}

Spread of automatic systems with significant reductions in management costs and possible increases in capacity. Increased urban and symbolic values of rail stations (the so-called Station Renaissance) could result in more attractiveness for these modes (Cascetta and Cartenì, 2012).

\subsubsection{Trams/medium capacity surface mass transit}

Development of semi-automatic or fully automatic systems allowing capacity increases and reduction in management costs.

\subsubsection{Bikes and e-bikes}

The use of bicycles as "pure" travel mode or as complementary mode (e.g. bike + train) is experiencing a significant come back not only in traditionally bike-oriented cities (UE, 2010). Similarly electrically powered bikes are expanding at very high rates and a compound growth rate of 7.5 percent per year is expected up to 2018 (PikeResearch, 2012).

\subsection{Innovations in ICT and Intelligent Transportation Systems}

\subsubsection{Personal information}

Widespread access to real time info on available transport options, including intermodal opportunities.

\subsubsection{System-wide monitoring and control}

Generalization of monitoring systems based on different date sources including individual and vehicles mobile communication devices, fixed infrastructure related technologies ( e.g. cameras) and others.

\subsubsection{Individual mobility pricing}

Spread of individual payment systems allowing more sophisticated pricing schemes, including mobility credits taking into account positive (e.g. walking or biking) and negative (e.g. drive-alone car in congested areas) modes or trip sections and possible compensations. 


\subsection{Mobility markets}

\subsubsection{Ownership vs. use of individual modes}

One of the relevant changes in this respect could be a transition from owning to using especially with respect to individual modes. If sustained this trend would open new markets for mobility services (fleet management, etc.) as well as for the auto industry (levels of demand and typology).

\subsubsection{Provision of intermediate mobility services}

Possible development of intermodal services including the availability of mixed individual/collective modes such as collective taxis, individual access to transit terminals, etc.

\subsubsection{Regulations and pricing of transportation services}

Spread of pricing structures and regulations allowing more energy/economy/environment efficient urban transportation. These could include systematic use-based road pricing and individual trip pricing (see section 4.1).

Opening of traditional (e.g. public transportation) and innovative mobility services to market competition.

\subsection{Technological innovation: new emergent modes}

\subsubsection{Individual/Collective mixed modes}

Introduction of vehicles acting as individual units in sparser parts of the road network and joining regulated "trains" of such vehicles for larger capacity in main haul sections.

\subsubsection{Autonomous driving vehicles}

Individual (cars) or collective (buses) vehicles driving autonomously based on vehicle-to-vehicle $(\mathrm{V} 2 \mathrm{~V})$ and vehicle-to-infrastructure (V2I) technologies on specialized sections of the road network increasing capacity and reducing management costs.

\subsection{City logistics and freight distribution}

\subsubsection{Technological innovation in distribution vehicles}

More specialized urban distribution vehicles including sizes, energy and pollution as well as pick-up/ delivery operations.

\subsubsection{Regulations and pricing}

Regulations limiting the distribution of freight via time window/vehicle type/load factors in order to reduce the increasing contribution to urban congestion connected to the likely increasing number of deliveries connected increase of e-commerce and reverse logistics. 


\section{Scenarios and possible evolution paths}

Obviously changes in the various factors listed above are not independent from each other. On the contrary there are strong correlations among some of the factors. For instance, a possible increase in fuel prices will probably reduce the demand for less energy efficient modes, increase the use and promote technological innovation of other modes, reduce available income and travel demand on the whole, and promote denser land use patterns. In this respect several internally consistent scenarios can be figured out based on consistent sets of assumptions on the key drives and related changes in other factors.

According to scenario building techniques (Shoemaker, 1995), variables should first be distinguished into important variables (i.e. variables offering the future but somehow predicable) and uncertain variables (i.e. elements subject to significant alternative outcomes).

In the following I'll hint some scenarios for illustrative purposes suggesting to adopt mixed scenarioDelphi method for the building of future scenarios of urban mobility (von der Gracht, 2010).

\subsection{Incremental scenario I: business as expected}

This scenario should represent the trends-as-they-are evolution of present urban mobility systems. This obviously implies changes with respect to present. In this scenario individual major changes in energy prices and availability, cars and individual modes will evolve technologically but will still the major urban mobility solutions for most cities in the world with increasing overall market as developing Countries motorization rates will approach those of developed ones. ITS technology and possibly reduced car sizes will help in providing capacity without major network extensions, at least in developed cities, while major highway constructions are to be expected in developing cities, especially in the BRICS Countries. Environmental requirements will either be eluded or complied with a mix of increased fuel efficiency, optimal congestion management and new energy sources.

Further, urban mobility changes will result more from local choices than from global factors. Some cities will evolve their mobility systems trying to optimize the use of individually owned cars while others will steer towards less car dependent systems based on livability as a competitive factor. Technologic evolutions will support both type of choices. It could also be possible than the same city will specialize parts of their territory according to different mobility schemes (e.g. car-less city centers as opposed to car-dependent peripheral areas). In this process the connection to High Speed Railways systems could influence cities specialization one way or another.

\subsection{Incremental scenario II: modal equilibration}

In this scenario still no significant technological breakthroughs, but the joined effects of increased energy prices and/or environmental concerns and/or space and life quality reclamation will push many cities towards policies aimed at modifying modal shares at least for certain trip types. This would probably require the implementation of demand management policies, possibly based on widespread use of pricing and mobility credits, investing in better transit, and mostly mass transit, systems, urban densification policies, the opening of transit and mobility services markets (e.g. car and bike sharing systems on larger scales), promotion of multimodal trips and chains for parts of the city. ITS will be part of the process with an emphasis on trip planning, system monitoring and automatic toll/credits collection. In several cities, walking/biking will increase their share of urban mobility.

\subsection{Revolution I: energy crisis}

In this scenario scarcity of traditional fuels and delays in new low cost energy sources will bring significant prices increases, and possibly limitations in the availability of fuels for individual modes, 
to a level making car as we know it no longer the basic option for urban mobility. The main difference with the modal equilibration scenario is that car reduced use will not result from a planned congestion reduction strategy, but from overall reduced availability. This will lower car shares and promote a number of changes in cities as well. It is likely that real estate markets will show major changes as peripheral areas will be less and less attractive, traditional and new mobility services, as trip planning, vehicles sharing, collective taxis will develop with the help of ITS technologies. Given this scenario urban mobility is going to be a major factor of competitiveness among cities, with those cities with well developed transit systems taking the edge.

\subsection{Revolution II: automation}

This scenario is prompted by technological innovation, especially in the field of individual vehicles. Cars, or their substitutes, will increasingly be able to run in dual mode. Drivers control and system control, with significant increases of system capacity and energetic efficiency. Vehicles will either be individually owned and operated, as evolution of present day cars, or operated by mobility service providers responding with autonomous driving vehicles to the needs of travelers on whole trips or at least for some trip sections (e.g. access to terminals, main roads, etc.). ITS developments will accordingly support the diffusion of autonomous driving, individual trip planning and system optimization. In this scenario energy prices will not be an issue either because traditional fuels will be available or because new low cost energy sources will be developed.

\section{References}

Kenworthy, J. R. (2006), The eco-city: ten key transport and planning dimensions for sustainable city development, Environment and Urbanization.

Cascetta, E. (2013), A new look at planning and designing transportation systems: markets, decisionmaking models and the role of quantitative methods, Transport Policy, under review.

Cascetta, E., and A. Cartenì (2013), The value of beauty in railways stations. A quantitative analysis of aesthetic quality on travelers behavior. Transportation Research Part A, under review.

Rogerson, R. J. (1999), Quality of Life and City Competitiveness, Urban Studies.

Banister, D. (1996), Energy, quality of life and the environment: the role of transport, Transport Reviews: A Transnational Transdisciplinary Journal, 16, 1.

Gilbert, R., and A. Perl (2010), Transport Revolutions: Moving People and Freight without Oil, New Society Publishers.

von der Gracht, H. A. , and I. L. Darkow (2010), Scenarios for the logistics services industry: A Delphi-based analysis for 2025, International Journal of Production Economics, 127,1.

Schoemaker, P. J. H. (1995), Scenario Planning: A Tool for Strategic Thinking, Sloan Management Review.

European Union Directorate General for Internal Affairs (2010), The promotion of cycling, Report. 